\title{
„Die Radiologie ist ein wichtiger Baustein in der Diagnostik bei Verdacht auf Kindesmisshandlung“
}

Das Bundeskriminalamt hat vor Kurzem Daten veröffentlicht, nach denen die Zahl misshandelter Kinder im Lockdown-Jahr 2020 um $10 \%$ gegenüber 2019 zugenommen hat. Mit Kindesmisshandlungen befassen sich auch die Radiologie und speziell die forensische Bildgebung. Wir haben mit der Radiologin Dr. Sarah Heinze etwa über die Frage gesprochen, wie Radiologen und Radiologinnen Kindesmisshandlungen erkennen können und welche, auch rechtlichen Schritte, sie einleiten müssen, wenn sie Misshandlungen feststellen. Dr. Heinze ist Fachärztin für Radiologie und Rechtsmedizin und als Leitende Oberärztin und Fachbereichsleiterin Forensische Bildgebung und Forschung am Institut für Rechts- und Verkehrsmedizin am Universitätsklinikum Heidelberg tätig.

Frau Doktor Heinze, wie stark sind Sie in Ihrem Arbeitsalltag mit dem Thema Kindesmisshandlung konfrontiert?

Leider fast jeden Tag. In unserem Forensic Radiology Center bekommen wir als Fachabteilung für forensische Bildgebung Konsilanfragen sowohl von umliegenden Kliniken, die wir im Rahmen unserer Gewaltambulanz versorgen, als auch von entfernteren Kliniken, teils bis hinunter zum Bodensee. Zusätzlich erhalten wir Gutachtenaufträge von Gerichten, um anhand vorhandener Krankenunterlagen und radiologischer Daten zu beurteilen, ob es sich um eine Kindesmisshandlung handelt und um Aussagen zur Lebensgefährlichkeit, zum Zeitpunkt der Entstehung und zur Mehrzeitigkeit der Verletzungen zu treffen.

Welche Verletzungen sind im Zuge von Misshandlungen aus Ihrer Erfahrung besonders häufig bei Kindern anzutreffen?

Überwiegend handelt es sich bei den nach Kindesmisshandlung festgestellten Verletzungen um Folgen stumpfer Gewalteinwirkung, beispielsweise nach Schlägen, Kneifen, Ohrfeigen, erzwungenem Füttern und ähnlichem. Auch Verbrühungen und Verbrennungen sind nicht selten, ebenso schwerere
Verletzungen wie Knochenbrüche, oft unterschiedlich alt. Wir sehen auch häufig Einblutungen in das Schädelinnere, zum Beispiel nach Schütteltrauma. Im Vergleich zu Erwachsenen sind Verletzungen nach scharfer Gewalt oder nach Strangulation hingegen selten.

\section{Welche Altersgruppe ist besonders be- troffen?}

Grundsätzlich kommen Kindesmisshandlungen in jedem Alter vor, gehäuft jedoch bei Kindern unter 4 Jahren. In dieser Gruppe betreffen viele Vorfälle das 1. Lebensjahr, in dem Kinder besonders vulnerabel sind.

Wie definieren sich Kindesmisshandlungen beziehungsweise wie können sie von anderen Verletzungen unterschieden werden?

Eine einheitliche Definition des Begriffs Kindesmisshandlung existiert nicht. Allgemein wird darunter jedoch jede Form der körperlichen, sexuellen oder psychischen Gewalt gegen Kinder verstanden. Diese kann durch aktives Tun oder Unterlassen entstehen. Die Abgrenzung misshandlungsbedingter Verletzungen von solchen, die im Rahmen des normalen kindlichen Verhaltens oder von Unfällen entstanden sind, erfordert hochspezialisierte Fachexpertise. Manche Befunde, wie zum Beispiel Doppelstriemen nach Schlägen mit länglichen Gegenständen oder bestimmte Frakturformen, sind sehr charakteristisch und praktisch beweisend für eine Misshandlung. In anderen Fällen ist eine sehr differenzierte morphologische Analyse unter Einbezug der konkreten Umstände im Einzelfall erforderlich, um eine sichere Unterscheidung treffen zu können.

Zu welchem Zeitpunkt beziehungsweise in welchen Versorgungssituationen kommt hier die Radiologie ins Spiel?

Zu verschiedenen Zeitpunkten. In der Klinik ist die Radiologie ein wichtiger Punkt in der Akutdiagnostik. Kommt zum Beispiel ein Kind mit einer Fraktur ins Krankenhaus, liegt es neben den Kinderärzten und Kin-

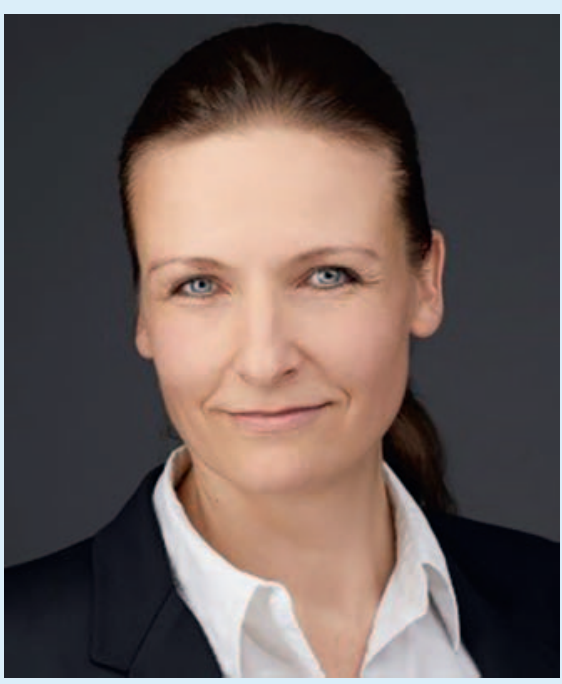

Dr. Sarah Heinze

derärztinnen an den Radiologen und Radiologinnen, anhand der Fraktur den Verdacht auf eine Kindesmisshandlung zu äußern und die radiologischen Bilder entsprechend zu befunden. In der forensischen Bildgebung sind wir im Rahmen von Konsilen und Untersuchungen der Gewaltambulanz eingebunden, wenn die Kinder gerade aufgenommen worden sind, und haben dann die Möglichkeit, noch aktiv in die Untersuchungen einzugreifen bzw. ergänzende Untersuchungen zu empfehlen. Später, wenn es bereits zu Verfahren gekommen ist, finden unter Umständen erst nach Monaten Nachbefundungen der Daten im Rahmen von schriftlichen Gutachten mit der Sachverständigentätigkeit vor Gericht statt.

Wie werden Kindesmisshandlungen in der Radiologie erkannt?

Hier darf ich freundlicherweise auf unser Webinar vom 17. Juni 2021 „Forensische Radiologie II - Battered Child“ auf dem 102. Deutschen Röntgenkongress verweisen. Dort haben wir typische radiologische Befunde bzw. spezifische Verletzungstypen bei Misshandlung vor- und die Schlüsselstellung der Radiologie in der diagnostischen Kaskade dargestellt sowie die Schwierigkeit der Diagnostik des Zeitpunkts der Verletzungsentstehung und der Mehrzeitigkeit diskutiert. 
Was sollten Radiologen und Radiologinnen zur Bildgebung bei Kindesmisshandlungen wissen?

Radiologen und Radiologinnen sollten die spezifischen Verletzungstypen bei Misshandlung und typische Differenzialdiagnosen kennen und sich mit der notwendigen und optimierten Bildgebung beschäftigen. So kann gewährleistet werden, dass, wenn Untersuchungen durchgeführt werden, diese auf die Erfassung von Misshandlungen optimiert sind und die Diagnostik so ermöglicht oder vervollständigt wird. Des Weiteren sollte man sich über die Notwendigkeit und den richtigen Zeitpunkt für Folgeuntersuchungen informieren. Am Herzen liegt mir zu erwähnen, dass jeder bei uns im Forensic Radiology Center jederzeit anfragen kann und wir mit Rat und Tat zur Seite stehen. Nicht jeder kann und muss ein Experte/eine Expertin auf dem Gebiet sein. Es ist wichtig zu wissen, wann man Hilfe benötigt und wo diese zu erhalten ist.

Könnten Sie uns ein leitliniengerechtes, radiologisches Vorgehen in diesem Bereich beschreiben?

Das leitliniengerechte Vorgehen hängt vom Alter des Kindes und von den Symptomen bzw. den Verletzungen ab. Für die Radiologie ist das Vorgehen sehr schön in dem Artikel von Mark Born et al. Die deutsche S3-Kinderschutzleitlinie - Bildgebung bei Verdacht auf Kindesmisshandlung (Fortschr Röntgenstr 2020; 192: 343-348) und natürlich in der AWMF S3 +-Kinderschutzleitlinie beschrieben. Auch die Kitteltaschenkarte, die man über die Internetseite der AWMF herunterladen kann, hilft als erster Überblick.
Wenn in der Radiologie die Misshandlung eines Kindes erkannt wird - welche Schritte, vielleicht auch rechtlicher Art, müssen Radiologen und Radiologinnen dann einleiten? Wie sieht der Prozess nach dem Erkennen aus?

Wie bereits erwähnt, ist die Radiologie ein wichtiger Baustein in der Diagnostik bei Verdacht auf Kindesmisshandlung. Wenn der Verdacht besteht, erfolgt die strukturierte Erfassung aller Angaben und Befunde interdisziplinär. Das Kind sollte in der Klinik aufgenommen werden, und je nach Alter und Verletzungen wird dann im 4-AugenPrinzip die weitere Diagnostik veranlasst. In der Radiologie umfasst diese strukturierte Diagnostik das Röntgen-Skelettscreening, die MRT von Schädel und Wirbelsäule, gegebenenfalls auch eine CT des Schädels sowie eventuelle weitere MRT-Untersuchungen und die Sonografie. Das Ziel ist es, die Anhaltspunkte zu objektivieren, die Einschätzung sicher zu vermitteln und eine (Entwicklungs-)Prognose zu erstellen. Insbesondere mit Hinblick auf den Aspekt, dass ein Wiederholungsrisiko (auch für eventuelle Geschwisterkinder) besteht, sollten Ärzte und Ärztinnen nicht zögern, neben dem Jugendamt auch die Strafverfolgungsbehörden einzuschalten.

Ist nach Ihrer Einschätzung die Sensibilität für das Thema in unserer Gesellschaft, aber auch im ärztlichen Kontext inzwischen im notwendigen Maß vorhanden oder besteht noch Informations- und Aufklärungsbedarf?

Obwohl die Sensibilität in der Gesellschaft in den letzten Jahren deutlich zugenommen hat und beispielsweise in Kindergärten, Schulen oder Kliniken wesentlich mehr auf das Thema geachtet wird, trifft die „gsunde Watschn" als Erziehungsmittel immer noch auf ein gewisses Verständnis. Auch hören wir als Rechtsmediziner und Rechtsmedizinerinnen vor Gericht häufig, dass man sich nicht vorstellen kann, dass das Kind von jemandem im engeren Umfeld misshandelt wurde. Teilweise wird dementsprechend immer noch weggesehen und nichts unternommen, wenn ein Verdachtsfall auftritt. Zudem fehlen nach wie vor verbindliche Regelungen hinsichtlich der Meldung solcher Fälle. Und auch die Umsetzung der existierenden fachlichen Standards, welche Maßnahmen nach Misshandlung erfolgen sollten, erfolgt nicht immer und überall gleich gut. Um die Kinder bestmöglich zu vertreten, bedarf es einer interdisziplinären $\mathrm{Zu}$ sammenarbeit mit gerichtsfester Dokumentation in jedem einzelnen Fall.

\section{INFORMATIONEN ZUM THEMA} KINDESMISSHANDLUNG

Nach kürzlich vom Bundeskriminalamt vorgestellten Zahlen verzeichnet die polizeiliche Kriminalstatistik für das Jahr 20204918 Fälle von Kindesmisshandlung - ein Anstieg um $10 \%$ gegenüber dem Vorjahr. 96,6\% der Fälle konnte die Polizei aufklären, doch diese Quote bezieht sich ausschließlich auf die angezeigten Fälle. Bei Kindesmisshandlung geht die Polizei von einer hohen Dunkelziffer aus, da die Taten im familiären Umfeld geschehen und die meisten betroffenen Kinder noch zu klein sind, um darüber zu sprechen. Ältere betroffene Kinder schweigen oft aus Scham. Befragungen von Jugendlichen und jungen $\mathrm{Er}$ wachsenen zeigen, dass etwa 5-10\% aller Eltern schwerwiegende und relativ häufig Körperstrafen bei ihren Kindern anwenden. 